Скопје, Македонија

\title{
SOME NEW FIXED POINT THEOREMS IN 2-BANACH SPACES
}

\author{
SAMOIL MALCHESKI, MARTIN LUKAREVSKI, AND KATERINA ANEVSKA
}

\begin{abstract}
S. Ghler ([9]), 1965, defined the 2-normed space, A. White ([3]), 1968, defined the 2-Banach space. Several statements about them are proven in [7]. P. K. Hatikrishnan and K. T. Ravindran in [5] defined the contractive mapping in 2-normed space. M. Kir and H. Kiziltunc in [3] by applying the above theorem, proved the generalizations of $\mathrm{R}$. Kannan ([6]) and S. K. Chatterjea ([10]) theorem. Further generalizations of these results are elaborated in [1] and [11]. In this paper we will generalize the above results by using the class $\Theta$ of monotony increasing functions $f:[0,+\infty) \rightarrow \mathbf{R}$ such that $f^{-1}(0)=\{0\}$ holds true.
\end{abstract}

\section{INTRODUCTION}

Theory of fixed point is rapidly developing last decades. S. Ghler ([9]) and A. White ([2]), 1965 and 1969, defined the 2-normed and 2-Banach spaces, and certain classical results about this theory, are generalized in 2-normed and 2-Banach spaces. P. K. Hatikrishnan and K. T. Ravindran defined contractive mapping in 2-normed space.

Definition 1. ([5]). Let $(L,\|\cdot, \cdot\|)$ be a real 2-normed space. Mapping $T: L \rightarrow L$ is said to be contraction if there exists $\lambda \in[0,1)$ such that $\|T x-T y, z\| \leq \lambda\|x-y, z\|$, for all $x, y, z \in L$, holds true.

P. K. Hatikrishnan and K. T. Ravindran in [5] proved that for each contractive mapping, a unique fixed point in closed and bounded subset of 2-Banach space $L$, exists.

M. Kir and H. Kiziltunc in [3] proved that if $S: L \rightarrow L$ satisfies one of the following conditions

$$
\|S x-S y, z\| \leq \lambda(\|x-S x, z\|+\|y-S y, z\|)
$$

for all $x, y, z \in L$

or

$$
\|S x-S y, z\| \leq \lambda(\|x-S y, z\|+\|y-S x, z\|)
$$

2000 Mathematics Subject Classification. 46J10, 46J15, 47H10.

Key words and phrases. 2-Banach spaces, fixed point, contraction mappings . 
for all $x, y, z \in L$ for $\lambda \in\left(0, \frac{1}{2}\right)$, then it exists a unique fixed point for $S$ in $L$. In [11] further generalizations for these results are proven, and in [1], by using a sequentially convergent mapping, are proved the generalized M.

Kir and H. Kiziltunc theorems. In our further considerations, by using the class $\Theta$ of monotony increasing continuous functions $f:[0,+\infty) \rightarrow \mathbf{R}$ such that $f^{-1}(0)=\{0\}$, we will elaborate the generalizations for some of the stated results. It is necessary to be said that if $f \in \Theta$, then $f^{-1}(0)=\{0\}$ implies that $f(t)>0$, for each $t>0$, holds true, and that the proves will be done by using the sequentially convergent, defined as the following:

Definition 2. Let $(L,\|\cdot, \cdot\|)$ be a 2-normed space. A mapping $T: L \rightarrow L$ is said sequentially convergent if we have, for every sequence $\left\{y_{n}\right\}$, if $\left\{T y_{n}\right\}$ is convergence then $\left\{y_{n}\right\}$ also is convergence.

\section{MAINS RESULTS}

Theorem 1. Let $(L,\|\cdot, \cdot\|)$ be a 2 -Banach space, $S: L \rightarrow L, f \in \Theta$ and $T: L \rightarrow L$ is continuous, injection and sequentially convergent mapping. If there exists $\alpha \in\left(0, \frac{1}{2}\right)$ such that

$$
f(\|T S x-T S y, z\|) \leq \alpha[f(\|T x-T S x, z\|)+f(\|T y-T S y, z\|)]
$$

for all $x, y, z \in L$, is satisfied, then for $S$ it exists a unique fixed point and for each $x_{0} \in X$ the sequence $\left\{S^{n} x_{0}\right\}$ converges to the above fixed point.

Proof. Let $x_{0}$ be any point on $L$ and the sequence $\left\{x_{n}\right\}$ be defined as the following $x_{n+1}=S x_{n}, n=0,1,2,3, \ldots$. The inequality (3) implies

$$
\begin{aligned}
f\left(\left\|T x_{n+1}-T x_{n}, z\right\|\right)= & f\left(\left\|T S x_{n}-T S x_{n-1}, z\right\|\right) \\
& \leq \alpha\left[f\left(\left\|T x_{n}-T S x_{n}, z\right\|\right)+f\left(\left\|T x_{n-1}-T S x_{n-1}, z\right\|\right)\right] \\
& \leq \alpha\left[f\left(\left\|T x_{n}-T x_{n+1}, z\right\|\right)+f\left(\left\|T x_{n-1}-T x_{n}, z\right\|\right)\right]
\end{aligned}
$$

So,

$$
f\left(\left\|T x_{n+1}-T x_{n}, z\right\|\right) \leq \lambda f\left(\left\|T x_{n}-T x_{n-1}, z\right\|\right)
$$

for each $n=0,1,2,3, \ldots$ and each $z \in L$, for $\lambda=\frac{\alpha}{1-\alpha}<1$. The inequality (4) implies

$$
f\left(\left\|T x_{n+1}-T x_{n}, z\right\|\right) \leq \lambda^{n} f\left(\left\|T x_{1}-T x_{0}, z\right\|\right)
$$

for each $n=0,1,2,3, \ldots$ and each $z \in L$. Further, (5) implies that for all $m, n \in \mathbf{N}, n>m$ and each $z \in L$

$$
\begin{aligned}
f\left(\left\|T x_{n}-T x_{m}, z\right\|\right)=f\left(\left\|T S x_{n-1}-T S x_{m-1}, z\right\|\right) \\
\leq \alpha\left[f\left(\left\|T S x_{n-1}-T x_{n-1}, z\right\|\right)+f\left(\left\|T S x_{m-1}-T x_{m-1}, z\right\|\right)\right] \\
=\alpha\left[f\left(\left\|T x_{n}-T x_{n-1}, z\right\|\right)+f\left(\left\|T x_{m}-T x_{m-1}, z\right\|\right)\right] \\
\leq \alpha\left[\lambda^{n-1} f\left(\left\|T x_{1}-T x_{0}, z\right\|\right)+\lambda^{m-1} f\left(\left\|T x_{1}-T x_{0}, z\right\|\right)\right] .
\end{aligned}
$$


holds true. The latter implies that

$$
\lim _{m, n \rightarrow \infty} f\left(\left\|T x_{n}-T x_{m}, z\right\|\right)=0,
$$

for each $z \in L$. And since $f \in \Theta$ we get that

$$
\lim _{m, n \rightarrow \infty}\left\|T x_{n}-T x_{m}, z\right\|=0,
$$

for each $z \in L$. Thus, the sequence $\left\{T x_{n}\right\}$ is Caushy, and since $L$ is 2Banach space, the sequence is convergent. Further, the mapping $T: L \rightarrow L$ is sequentially convergent, and therefore the sequence $\left\{x_{n}\right\}$ is convergent, i.e. it exists $u \in L$ such that $\lim _{n \rightarrow \infty} x_{n}=u$, holds true. The continuous of $T$ implies that $\lim _{n \rightarrow \infty} T x_{n}=T u$. So, for each $z \in L$

$$
\begin{aligned}
f\left(\left\|T S u-T x_{n+1}, z\right\|\right)= & f\left(\left\|T S u-T S x_{n}, z\right\|\right) \\
\leq & \alpha\left[f(\|T S u-T u, z\|)+f\left(\left\|T S x_{n}-T x_{n}, z\right\|\right)\right] \\
& =\alpha\left[f(\|T S u-T u, z\|)+f\left(\left\|T x_{n+1}-T x_{n}, z\right\|\right)\right],
\end{aligned}
$$

holds true. For $n \rightarrow \infty$, the continuous of $f$ and $T$ and the properties of 2-norm imply that

$$
f(\|T S u-T u, z\|) \leq \alpha[f\|T S u-T u, z\|)+f(0)],
$$

for each $z \in L$, holds true. But, $\alpha \in(0,1)$ and $f^{-1}(0)=\{0\}$, therefore, the latter inequality implies that $\|T S u-T u, z\|=0$, for each $z \in L$, that is $T S u=T u$. Finally, $T$ is injection, therefore $S u=u$, that is it exists a fixed point for $S$. Let $u, v \in X$ be two fixed points for $S$, i.e. $S u=u$ and $S v=v$. Then (3) implies that

$$
\begin{aligned}
f(\|T u-T v, z\|)= & f(\|T S u-T S v, z\|) \\
\leq & \alpha[f(\|T u-T S u, z\|)+f(\|T v-T S v, z\|)] \\
& =0,
\end{aligned}
$$

for each $z \in L$, that is $\|T u-T v, z\|=0$, for each $z \in L$, i.e. $T u=T v$. But, since $T$ is injection, we get that $u=v$, that is $T$ has a unique fixed point. Finally, the arbitrarily of $x_{0} \in L$ and the above stated, imply that for each $x_{0} \in L$ the sequence $\left\{S^{n} x_{0}\right\}$ converges to the unique fixed point for $S$.

Consequence 1. Let $(L,\|\cdot, \cdot\|)$ be a 2-Banach space, $S: L \rightarrow L$, $f \in \Theta$ and $T: L \rightarrow L$ is continuous, injection and sequentially convergent mapping. If there exists $\lambda \in(0,1)$ such that

$$
f(\|T S x-T S y, z\|) \leq \lambda \sqrt{f(\|T x-T S x, z\|) f(\|T y-T S y, z\|)}
$$

for all $x, y, z \in L$, holds true, then $S$ has a unique fixed point. 
Proof. The proof is directly implied by the arithmetic-geometric inequality mean and the Theorem 1 , for $\alpha=\frac{\lambda}{2}$.

Consequence 2. Let $(L,\|\cdot, \cdot\|)$ be a 2-Banach space, $S: L \rightarrow L, f \in \Theta$. If there exists $\alpha \in\left(0, \frac{1}{2}\right)$ such that

$$
f(\|S x-S y, z\|) \leq \alpha[f(\|x-S x, z\|)+f(\|y-S y, z\|)],
$$

for all $x, y, z \in L$, holds true, then $S$ has a unique fixed point and for each $x_{0} \in X$ the sequence $\left\{S^{n} x_{0}\right\}$ converges to that fixed point.

Proof. The mapping $T x=x$, for each $x \in L$ is continuous, injection and sequentially convergent. The statement is directly implied by the Theorem 1 for $T x=x$.

Consequence 3. ([1]). Let $(L,\|\cdot, \cdot\|)$ be a 2 -Banach space, $S: L \rightarrow$ $L$ and $T: L \rightarrow L$ is continuous, injection and sequentially convergent mapping. If there exists $\alpha \in\left(0, \frac{1}{2}\right)$ such that

$$
\|T S x-T S y, z\| \leq \alpha(\|T x-T S x, z\|+\|T y-T S y, z\|)
$$

for all $x, y, z \in L$, holds true, then $S$ has a unique fixed point and for each $x_{0} \in X$ the sequence $\left\{S^{n} x_{0}\right\}$ converges to that fixed point.

Proof. The function $f(t)=t, t \geq 0$ is monotony increasing and $f^{-1}(0)=\{0\}$. The statement is directly implied by Theorem 1 for $f(t)=t$.

Remark 1. For $f(t)=t, t \geq 0$ (1) is implied by (6), that is Theorem 1, [3] is implied by Consequence 2 .

Theorem 2. Let $(L,\|\cdot, \cdot\|)$ be a 2-Banach space, $S: L \rightarrow L, f \in \Theta$ and $T: L \rightarrow L$ is continuous, injection and sequentially convergent mapping. If there exists $\alpha \in\left(0, \frac{1}{2}\right)$ such that

$$
f\left(\|T S x-T S y, z\|^{2}\right) \leq \alpha\left[f\left(\|T x-T S x, z\|^{2}\right)+f\left(\|T y-T S y, z\|^{2}\right)\right]
$$

for all $x, y, z \in L$, holds true, then $S$ has a unique fixed point and for each $x_{0} \in X$ the sequence $\left\{S^{n} x_{0}\right\}$ converges to that fixed point.

Proof. Let $x_{0}$ be any point in $L$ and the sequence $\left\{x_{n}\right\}$ be defined as the following $x_{n+1}=S x_{n}, n=0,1,2,3, \ldots$ The inequality (7), analogously as the Proof of Theorem 1, implies that

$$
f\left(\left\|T x_{n+1}-T x_{n}, z\right\|^{2}\right) \leq \lambda f\left(\left\|T x_{n}-T x_{n-1}, z\right\|^{2}\right)
$$

for each $n=0,1,2,3, \ldots$ and $z \in L$, for $\lambda=\frac{\alpha}{1-\alpha}<1$, holds true. Further, by applying (8) analogously as the proof of Theorem 1 , the sequence $\left\{x_{n}\right\}$ is convergent, i.e. it exists $u \in L$ such that $\lim _{n \rightarrow \infty} x_{n}=u$. Since $T$ is continuous, we get that $\lim _{n \rightarrow \infty} T x_{n}=T u$. The inequality (7), analogously as the proof of Theorem 1 , we get that

$f\left(\left\|T S u-T x_{n+1}, z\right\|^{2}\right) \leq \alpha\left[f\left(\|T S u-T u, z\|^{2}\right)+f\left(\left\|T x_{n+1}-T x_{n}, z\right\|^{2}\right)\right]$, holds true. For $n \rightarrow \infty$, the latter is transformed as the following

$$
\left.f\left(\|T S u-T u, z\|^{2}\right) \leq \alpha\left[f\|T S u-T u, z\|^{2}\right)+f(0)\right],
$$


for each $z \in L$. Analogously, as the ?f Theorem 1 we get that $S u=u$, that is $S$ has a fixed point. Let $u, v \in X$ be fixed points for i.e. $S u=u$ and $S v=v$. Then, (7) implies

$$
\begin{aligned}
f\left(\|T u-T v, z\|^{2}\right)= & f\left(\|T S u-T S v, z\|^{2}\right) \\
& \leq \alpha\left[f\left(\|T u-T S u, z\|^{2}\right)+f\left(\|T v-T S v, z\|^{2}\right)\right] \\
& =0,
\end{aligned}
$$

for each $z \in L$. Therefore, we get that $u=v$, i.e. $S$ has a unique fixed point. Finally, the arbitrarily of $x_{0} \in L$ and the above stated, imply that for each $x_{0} \in L$ the sequence $\left\{S^{n} x_{0}\right\}$ converges to the unique fixed point for $S$.

Consequence 4. Let $(L,\|\cdot, \cdot\|)$ be a 2 -Banach space, $S: L \rightarrow L$ and $f \in \Theta$. If there exists $\alpha \in\left(0, \frac{1}{2}\right)$ such that

$$
f\left(\|S x-S y, z\|^{2}\right) \leq \alpha\left[f\left(\|x-S x, z\|^{2}\right)+f\left(\|y-S y, z\|^{2}\right)\right]
$$

for all $x, y, z \in L$, then $S$ has a unique fixed point and for each $x_{0} \in X$ the sequence $\left\{S^{n} x_{0}\right\}$ converges to that fixed point.

Proof. For $T x=x$ in Theorem 2, we get the given statement.

Consequence 5. ([1]). Let $(L,\|\cdot, \cdot\|)$ be a 2-Banach space, $S: L \rightarrow L$ and a mapping $T: L \rightarrow L$ is such that it is continuous, injection and sequentially convergent mapping. If there exists $\alpha \in\left(0, \frac{1}{2}\right)$ such that

$$
\|T S x-T S y, z\|^{2} \leq \alpha\left(\|T x-T S x, z\|^{2}+\|T y-T S y, z\|^{2}\right),
$$

for all $x, y, z \in L$, holds true, then there is a unique fixed point for $S$ and for each $x_{0} \in X$ the sequence $\left\{S^{n} x_{0}\right\}$ converges to the fixed point.

Proof. For $f(t)=t$ in Theorem 2, we get the given statement.

Remark 2. For $f(t)=t, t \geq 0$ the condition (9) in Consequence 4 is transformed as the following

$$
\|S x-S y, z\|^{2} \leq \alpha\left[\|x-S x, z\|^{2}+\|y-S y, z\|^{2}\right],
$$

that is Consequence 7, [1] is implied by Consequence 4 .

Theorem 3. Let $(L,\|\cdot, \cdot\|)$ be a 2 -Banach space, $S: L \rightarrow L$ and a mapping $T: L \rightarrow L$ be such that it is continuous, injection and sequentially convergent mapping and $f \in \Theta$ be such that $f(a+b) \leq f(a)+f(b)$, for all $a, b \geq 0$. If there exists $\alpha \in\left(0, \frac{1}{2}\right)$ such that

$$
f(\|T S x-T S y, z\|) \leq \alpha[f(\|T x-T S y, z\|)+f(\|T y-T S x, z\|)]
$$

holds true, then there is a unique fixed point for $S$ and for each $x_{0} \in X$ the sequence $\left\{S^{n} x_{0}\right\}$ converges to the fixed point.

Proof. Let $x_{0}$ be any point in $L$ and the sequence $\left\{x_{n}\right\}$ be defined as the following $x_{n+1}=S x_{n}, n=0,1,2,3, \ldots$. The inequality (10) and the 
properties of $f$ imply

$$
\begin{aligned}
f\left(\left\|T x_{n+1}-T x_{n}, z\right\|\right) & =f\left(\left\|T S x_{n}-T S x_{n-1}, z\right\|\right) \\
\leq & \alpha\left[f\left(\left\|T x_{n}-T S x_{n-1}, z\right\|\right)+f\left(\left\|T x_{n-1}-T S x_{n}, z\right\|\right)\right] \\
= & \alpha f\left(\left\|T x_{n-1}-T x_{n+1}, z\right\|\right) \\
\leq & \alpha f\left(\left\|T x_{n-1}-T x_{n}, z\right\|+\left\|T x_{n}-T x_{n+1}, z\right\|\right) \\
\leq & \alpha\left[f\left(\left\|T x_{n-1}-T x_{n}, z\right\|\right)+f\left(\left\|T x_{n}-T x_{n+1}, z\right\|\right)\right] .
\end{aligned}
$$

So,

$$
f\left(\left\|T x_{n+1}-T x_{n}, z\right\|\right) \leq \lambda f\left(\left\|T x_{n}-T x_{n-1}, z\right\|\right),
$$

for each $n=0,1,2,3, \ldots$ and each $z \in L$, for $\lambda=\frac{\alpha}{1-\alpha}<1$. Analogously, as the proof of Theorem 1, we conclude that the sequence $\left\{x_{n}\right\}$ is convergent, i.e. it exists $u \in L$ such that $\lim _{n \rightarrow \infty} x_{n}=u$ and $\lim _{n \rightarrow \infty} T x_{n}=T u$. Further, for each $z \in L$

$$
\begin{aligned}
f\left(\left\|T S u-T x_{n+1}, z\right\|\right)= & f\left(\left\|T S u-T S x_{n}, z\right\|\right) \\
\leq & \alpha\left[f\left(\left\|T u-T S x_{n}, z\right\|\right)+f\left(\left\|T x_{n}-T S u, z\right\|\right)\right] \\
& =\alpha\left[f\left(\left\|T u-T x_{n+1}, z\right\|\right)+f\left(\left\|T x_{n}-T S u, z\right\|\right)\right],
\end{aligned}
$$

holds true. For $n \rightarrow \infty$, the continuous of $f$ and $T$ and the properties of 2-norm imply that $f(\|T S u-T u, z\|) \leq \alpha[f\|T S u-T u, z\|)+f(0)]$, for each $z \in L$, holds true. Now, analogously as the proof of Theorem 1 , we get that $S u=u$, that is it exists a unique fixed point for $S$. Let $u, v \in X$ be two fixed point for $S$, i.e. $S u=u$ and $S v=v$. Then (3) implies that the following

$$
\begin{aligned}
f(\|T u-T v, z\|)= & f(\|T S u-T S v, z\|) \\
\leq & \alpha[f(\|T u-T S v, z\|)+f(\|T v-T S u, z\|)] \\
& =2 \alpha f(\|T u-T v, z\|),
\end{aligned}
$$

for each $z \in L$, holds true. And since $2 \alpha<1$ we get that $\|T u-T v, z\|=0$, for each $z \in L$, holds true. The latter implies that $u=v$, that is there exists a unique fix point for $S$.

Consequence 6. Let $(L,\|\cdot, \cdot\|)$ be a 2 -Banach space, $S: L \rightarrow L$, the mapping $T: L \rightarrow L$ be such that it is continuous, injection and sequentially convergent mapping and function $f \in \Theta$ be such that $f(a+b) \leq f(a)+f(b)$, for all $a, b \geq 0$. If there exists $\lambda \in(0,1)$ so that

$$
f(\|T S x-T S y, z\|) \leq \lambda \sqrt{f(\|T x-T S y, z\|) f(\|T y-T S x, z\|)},
$$

for all $x, y, z \in L$, holds true, then there is a unique fixed point for $S$. 
Proof. The proof is directly implied by the arithmetic-geometric mean inequality and Theorem 3 , for $\alpha=\frac{\lambda}{2}$.

Consequence 7. Let $(L,\|\cdot, \cdot\|)$ be a 2 -Banach space, $S: L \rightarrow L$ and $f \in \Theta$ be such that $f(a+b) \leq f(a)+f(b)$, for all $a, b \geq 0$. If there exists $\alpha \in\left(0, \frac{1}{2}\right)$ so that

$$
f(\|S x-S y, z\|) \leq \alpha[f(\|x-S y, z\|)+f(\|y-S x, z\|)],
$$

for all $x, y, z \in L$, holds true, then there is a unique fixed point for $S$ and for each $x_{0} \in X$ the sequence $\left\{S^{n} x_{0}\right\}$ converges to that fixed point.

Proof. For $T x=x$ in Theorem 3, we get the given statement.

Consequence 8. ([1]). Let $(L,\|\cdot, \cdot\|)$ be a 2 -Banach space, $S: L \rightarrow L$ and the mapping $T: L \rightarrow L$ be such that it is continuous, injection and sequentially convergent. If there exists $\alpha \in\left(0, \frac{1}{2}\right)$ so that

$$
\|T S x-T S y, z\| \leq \alpha(\|T x-T S y, z\|+\|T y-T S x, z\|),
$$

for all $x, y, z \in L$, holds true, then there is a unique fixed point for $S$ and for each $x_{0} \in X$ the sequence $\left\{S^{n} x_{0}\right\}$ converges to that fixed point.

Proof. For $f(t)=t$ in Theorem 2, we get the given statement.

Remark 3. For $f(t)=t, t \geq 0$ (1) is implied by (6), that is Theorem 1 , $[3]$ is implied by Consequence 2 .

\section{CONFLICT OF INTEREST}

No conflict of interest was declared by the authors.

\section{AUTHORS CONTRIBUTIONS}

All authors contributed equally and significantly to writing this paper. All authors read and approved the final manuscript.

\section{REFERENCES}

[1] A. Malceski, A. Ibrahimi, R. Malceski, Extending Kannan and Chatterjea Theorems in 2-Banach Spaces by using Sequentialy Convergent Mappings, Matematicki bilten, Vol. 40, No. 1, pp. 29-36

[2] A. White, 2-Banach Spaces, Math. Nachr. Vol. 42 (1969), 43-60

[3] M. Kir, H. Kiziltunc, Some New Fixed Point Theorems in 2-Normed Spaces, Int. Journal of Math. Analysis, Vol. 7 No. 58 (2013), 2885-2890

[4] P. Chouhan, N. Malviya, Fixed Points of Expansive Type Mappings in 2-Banach Spaces, International Juornal of Analysis and Applications, Vol. 3 No. 1 (2013), 60-67

[5] P. K. Hatikrishnan, K. T. Ravindran, Some Properties of Accretive Operators in Linear 2-Normed Spaces, International Mathematical Forum, Vol. 6 No. 59 (2011), 2941-2847

[6] R. Kannan, Some results on fixed points, Bull. Calc. Math. Soc. Vol. 60 No. 1, (1968), 71-77

[7] R. Malceski, K. Anevska, About the 2-Banach spaces, International Journal of Modern Engineering Research (IJMER), Vol. 4 Iss. 5 (2014), 28-32 
[8] S. Banach, Sur les operations dans les ensembles abstraits et leur application aux equations intgrales, Fund. Math. 2 (1922), 133-181

[9] S. Ghler, Lineare 2-normierte Rume, Math. Nachr. 28 (1965), 1-42

[10] S. K. Chatterjea, Fixed point theorems, C. R. Acad. Bulgare Sci., Vol. 25 No. 6 (1972), 727-730

[11] R. Malceski, A. Ibrahimi, Contraction Mappings and Fixed Point in 2-Banach Spaces, IJSIMR, e-ISSN 2347-3142, p-ISSN 2346-304X, Vol. 4, Iss. 4, (2016), pp. 34-43

[12] M. Lukarevski and S. Malceski, Sequentuqlly Convergent Mappings and Common Fixed Points of Mappings in 2-Banach Spaces, Matematicki bilten, Vol. 40, No. 3, pp. 13-22

[13] R. Malceski, A. Malceski, K. Anevska and S. Malceski, Common Fixed Points of Kannan and Chatterjea Types of Mappings in a Complete Metric Space, British Journal of Mathematics \& Computer Science, Vol. 18 No. 2 (2016), 1-11

[14] A. Malceski, S. Malceski, K. Anevska and R. Malceski, New Extension of Kannan and Chatterjea Fixed Point Theorems on Complete Metric Spaces, British Journal of Mathematics \& Computer Science, Vol. 17 No. 1 (2016), 1-10

[15] R. Malceski, K. Anevska, About the 2-Banach spaces, International Journal of Modern Engineering Research (IJMER), Vol. 4 Iss. 5 (2014), $28-32$

University Euro-Balkan, Skopje, Macedonia

E-mail address: samoil.malcheski@gmail.com

Faculty of informatics, University Goce Delchev, Shtip, Macedonia

E-mail address: martin.lukarevski@ugd.edu.mk

Faculty of informatics, FOn University, Skopje, Macedonia.

E-mail address: anevskak@gmail.com 